2.

\title{
Ueber einen Fall von links verlanfender Vena cava superior, muthmaasslich bedingt durch frïhzeitige Synostose der Sutura mastoidea dextra.
}

\author{
Von Prof. Carl Weigert (Leipzig).
}

Im 81. Bande dieses Archivs (S. 458) ist von Wenzel Gruber eln Fall von links verlaufender Vena cava superior veröffentlicht worden, der der „dritte seit 167 Jahren bekannte" (elgentlich wohl der zweite, da der erste Fall sehr ungenügend beschrieben war) ist. Ich erlaube mir in Folgendem den vierten derartigen Fall zu veröffentlichen, der, abgesehen von der Seltenheit dieser Affection, noch dadurch interessant sein dürfte, weil er einen Ejablick in die Genese dieser sonderbaren Missbildung zu gewähren scheint, und zwar nach einer Richtung hin, welche bel den bisher bekannten Fällen nleht berücksichtigt worden ist. Andererseits dürfte es wohl dabei nichts verschlagen, dass der Verlauf einiger untergeordneter Venenäste nicht verfolgt werden konnte; es musste eben in erster Linie dem klinischen Interesse dieses Falles (Pseudoleukämie) Rechnung getragen werden.

Es handelte sich um elnen 26 jährigen kräftig gebauten Mann, bei dem sich beträchtliche Lymphome in der llnken Hals - und Achselgegend, in der rechten Lelstengegend etc. fanden, ferner eine sebr beträchtliche Milzanschwellung mit reichllch eingelagerten, zum Theil coagulationsnekrotisch untergegangenen Knoten, Lymphomknoten im Knochenmark, der linken Nebenniere, Thrombosen in Venen der unteren Extremitäten, Embolien mit Infarctbildungen in der Lunge, eine frische Endocarditis mitralis und von Missbildungen (mit Ausnabme der gleich noch näher zu beschreibenden) ein Meckel'sches Dünndarmdivertikel. - Bei diesem Manne springt nach Eröffoung des Herzbeutels ein grosser Venenstamm in die Augen, der yor den übrigen Gefässen an der Vorderseite des Herzens herabsteigt.

Diese grosse Vene setzt sich, noch bevor sie in den Herzbeutel eintritt, aus 3 grossen Stämmen zusammen; der grösste von diesen 3 Stämmen ist die unmittelhare Fortsetzung der Vena jug. sin., so dass der grosse Venenstamm innerhalb des Herzbentels durchaus in der Flucht dieser Vene liegt. Am Eingange des Thorax hat die Vene einen Umfang von $2,2 \mathrm{Gm}$., sie nimmt nun eine pon rechts herkommende grosse Vene auf, die einen Umfang ron $1,7 \mathrm{~cm}$. hat und der Anonyma dextra entspricht. $2 \mathrm{Cm}$. nach abwärts tritt von links her noch eine Vene von $2 \mathrm{Cm}$. Umfang an den Hauptstamm heran, welch' letaterer nunmehr bereits einen Umfang von $3,5 \mathrm{Cm}$. erreicht hat, - Diese von links herkommende Vene hat an ibrer Einmündungsstelle 2 Klappen, sie entspricht der Vena subclavia sin. [Es muss bemerkt werden, dass gerade um die Vena jug. int. sin. herum, bis zur Vena subclav. herab, ein ganz colossales Paquet geschwollener Lymphdrüsen sich findet, welches auch die Carotis sin. von der Jug. int. sin. abdrängt.] 
Was den Verlauf des grossen Venenstammes anbetrifft, der dle Fortsetzung der Vena jug. int. sin. darstellt, so liegt dieser Venenstamm vor allen übrigen grossen Gefässen und zwar speciell vor der Art. subclav. sin. Die Vena subclav. sin. tritt erst in der Höhe des Aortenbogens an den Hauptstamm heran, welcher ausser den genannten grossen Venen an seiner Hinterfläche noch an der Vorderfiäche je zwei kleinere Aestchen aufnimmt. Von den übrigen grossen Venen sei bemerkt, dass die Vena jug. int. dextr., sowie eine Vena cava sup. dextra vollkommen fehlt, während die Vena azygos dextra und die Vena jug. externa dextra als ein grosses Gefäss vorbanden ist.

Unterbalb der Einmündungsstelle der Vena subclav. sin. tritt nun jener grosse Venenstamm in den Herzbeutel ein, wobei er vor der Art. pulmonalis und zwar links vor ihrem Anfangstheile, dann auf den linken Pulmonalvenen, an der linken Wand der Auricula sin. sich haltend, zum Sulcus atrioventricularis herantritt, der den recbten Rand der grossen Vene bis zu ibrer Einmündung in das Herz bildet. $\mathrm{Da}$, wo der Venenstamm den oberen Rand der Auricula sin. erreicht, treten in seiner Wand rothe Muskelfasern auf. Die Vene mündet dann in den rechten Vorhof ein, und zwar in der Weise, dass sie sich nach binten und etwas nach rechts um den linken Vorhof herumschlägt. Die Einmündungsstelle in den Vorhof ist derartig, dass díeselbe unmittelbar benachbart, resp. etwas links von der Stelle sich findet, wo der linke Rand der Einmündung der Vena cava inf. ist. Die dem Herzen zugekebrte (rechte) Hälfte der Vene setzt sich dabei nnmittelbar in die Flucht des linken Zipfels der Valvula tricuspidalis fort. Bei ihrem Verlaufe längs des Sulcus coronar. cordis bedeckt sie die ganze rechte Hälfte der Vorderwand des linken Vorhofes, und ist, namentlich - vorn, überall mit deutlich rothen Muskelfasern versehen. Der Umfang der Vene unmittelbar vor ihrer Einmündung beträgt fast $7 \mathrm{Cm}$.

Alle diejenigen Herzvenen, welche sich sonst in den Sinus coron. ergiessen, münden hier in denjenigen Theil der Vena cava sup. sin., der längs des Sulcus atrioventric. verläuft. Zunächst findet sich eine grosse Vena coron. magna, die mit einem $2 \mathrm{Cm}$. langen Querstücke unterhalb der Auricula sin. herumläuft und in den rechten Rand der Vena cava sup. sin. mündet, dann ist eine Vena posterior ventr. sin. vorhanden und eine etwas kleinere Vena cordis media, die ebenfalls an den rechten Rand der Vena cava sup. sin. herantreten. An den linken Rand tritt eine Anzahl ganz feiner Venenästchen vom linken Theile des Atrium sin. an den Hauptstamm direct heran. In den Vorhof selbst ergiesst sich ausserdem noch eine Anzahl Venen, die nicht mit dem Hauptstamm in Verbindung treten, namentlich ist hier eine grosse Vena cordis Galeni zu bemerken.

Die Einmündungsstelle der Vena cava sup. ist klappenlos. Die Fossa ovalis ist klein $(0,6 \mathrm{Cm}$. im Durchmesser). Das For. ovale geschlossen. Das rechte Herzohr sehr weit. An den übrigen Theilen des Herzens, sowie an der Vena cava inf. und den Arterien sind keine besonderen Bildungsanomalien zu constatiren.

Maasse des Herzens: Dicke des linken Ventrikels in der Mitte 1,2 Cm. Höhe 9,5 Cm. Dícke des Conus dexter 0,4 Cm. Umfang des Anfangstheiles der Pulmonalis 7,0 $\mathrm{Cm}$. Umfang der Aorta descendens $4,5 \mathrm{Cm},-$

Bemerkenswerthe Anomalien finden sich jedoch auch in der Schädelhöhle vor. Während der Sin. transvers. sin. sehr weit ist und mit einer sehr weiten 0effaung 
in die Vena jug. interna sin. einmündet, ist der Sin. transv. dexter bedeutend kleiner; seine Mündung nach aussen gerade noch für eine dicke Sonde durchgăngig. Dle Verbindung dieser Stelle mit dem Gebiete der Vena jug. ext. dextra konnte, da die Leiche noch geschont werden musste, uicht weiter verfolgt werden. Hingegen gelang es nachträglich noch den Schädel zu bekommen, nach dessen Maceration sich Folgendes ergab:

Die Schädelbasis ist von in n e n betrachtet nicht ganz symmetrlsch. Die Mittellinie weicht ein wenig nach links ab. Die mittlere und vordere Schädelgrube rechts etwas flacher, als die der linken Seite. Die hintere rechte Schädelgrube hingegen schmäler als die linke. Der Sulcus sagit. setzt sich in den linken Schenkel des Sulcus transversus fort; der rechte Sulcus transvers. ist flacher und schmāler $(0,7: 1,1)$ als der linke; vor allem aber fällt auf, dass die Naht zwischen dem Seitentheil der Hinterhauptschuppe und dem Processus mastoides nur in ihrem ohersten Theile $1,5 \mathrm{Cm}$. weit rechts existirt, während der untere Theil derselben vollkommen fehlt, im Gegensatz zu der linksseitigen entsprechenden Naht, die wohl ausgebildet vorhanden ist. In dem Winkel zwischen Scheitelbein, Hinterhauptsschuppe und Warzentheil des Schläfenbeins sind beiderseits einige kleine Zwischenknochen vorhanden. Vom Sulcus transversus dexter geht im oberen Theile vom hinteren Rande aus ein $3 \mathrm{Mm}$. weites Foramen mastoideum nach hinten und aussen, im unteren Theile ein für eine dicke Sonde durchgängiger Canalis condyloideus gerade nach hinten. Das Foramen jugulare rechts ist durch eine Knochenspange in zwei Theile getheilt, von welchen der hintere (für die Vene bestimmte) ein wenig grösser als der vordere, von den Nerven eingenommene, ist. Links fehlt diese Knochenspange ebenso wie ein grösseres Foramen mastoideum und ein Canalis condyloideus. Zieht man von dem gemeinsamen Foramen jug. dextr. einen dem vorderen Theil rechts entsprechenden (für den Nerven bestimmten) Abschnitt ab, so ist der hintere venöse Theil rechts der Schätzung nach etwa ein Drittel so gross, als der venöse Theil links sein würde.

Bei Betrachtung des Schädels von a ussen zeigt sich eine erhebliche Asynmetrie in der Verbindungslinie der beiden Processus mastoides. Während die mediale Fläche des linken Processus mastoides von der Verbindungsstelle der Crista occipitalis mit dem Hinterhauptsloche, die genau median liegt, 5,6 Cm. entfernt ist, beträgt die entsprechende Entfernung rechts nur 4,7 $\mathrm{cm}$. Diese Verkleinerung der Entfernung kommt aber durchaus auf die Distanz zwischen Proc. mastoides und Linea semicircularis inferior, indem die unteren Enden beider Lineae semicirculares beiderseits $3,8 \mathrm{Cm}$, von der Mittellinle entfernt sind, so dass demnach die Entfernung zwischen Medianfläche des Proc. mastoides und unterem Theile der Linea semicircularis inferior rechts 0,9 , links $1,8 \mathrm{Cm}$. misst. Auch von aussen sieht man, dass rechts die Sutura mastoidea grösstentheils fehlt, an der Aussenfiäche bemerkt man im Gegensatz zur Innenfläche sogar nur eine eingezackte tiefe Rinne, welche vom Aussenrande des Foramen styloideum her $1,5 \mathrm{~cm}$. nach oben reicht, und vielleicht als Rest der Sutur anzusehen ist. Im Foramen jugulare selbst ist keine Spur einer Naht zu entdecken. Ebenso ist auch der Processus jugularis rechts ohne Naht mit den benachbarten Knochentheilen verbunden. An den übrigen Theilen des Schädels ist eine Abweichung nicht zu bemerken. 
Es handelt sich also um elne Missbildung, bei welcher die Vena cava sup. links und zwar vor dem Herzen herabsteigt, wobei ihr unteres Ende dem Sinus coronarius entspricht, ferner um ein Fehlen der Venajug. int. dextra, eine Aplasie des Sin. transversus dexter, eine synostose des rechten Seitentheils des 0s occipitis und der Pars mastoidea des rechten Schläfenbeins, mit Aplasie der in der Umgebung dieser Synostose gelegenen Knochentheile.

Es muss ausdrücklich hervorgehoben werden, dass das Fehlen der Vena jugularis interna destra in keiner Beziehung zo der Pseudolenkämie stehen kann, da gerade an der rechten Halsseite keine geschwollenen Lymphdrüsen vorhanden waren.

Die Erk]ärung für das Zustandekommen einer Vena cava sup. sin. an Stelle der dextra ist schon von Halbertsma gegeben. Sie findet sich namentlich sehr klar auseinandergesetzt bei Krause (Henle's Anatomie, "Varietäten der Venen“. Vgl. auch Kölliker's Entwickelungsgeschichte S. 928 ff.). Es handelt sich kurz gefasst um Folgendes: Ursprünglich bestehen 2 grössere Venenstämme für die obere Körperabtheilung, ein rechter und ein linker, die sich durch ein Verbindungsstück (Vena jugularis transversa) mit einander in Communication setzen. Von diesen beiden Stämmen, deren oberer Theil zu den Venae jugulares externae wird, persistirt nach dem 4. Embryonalmonate für gewöhnlich nur der rechte Venenstamm (Vena jugularis primitiva und Ductus Cuvieri dexter) vollständig, und bildet die Jugul. ext. dextra, die V. anonyma dextra und die gewöhnliche Vena cava sup., ferner bleibt das Verbindungsstäck erhalten and wird zur Vena anonyma sin.; von dem linken Venenstamme erhält sich (ausser der Vena jugul. externa sinistra) nur der dem Herzen unmittelbar anlegende Theil der Vene, d.h. der Sin. coronarius, während der Theil zwischen dem Sin. coron. und den Venae jugulares entweder ganz untergeht, oder ausnahmsweise als unbedeutender Venenast fortbesteht. Die Venae jugulares in ternae sind erst secundăre Bildungen, im Gegensatz zo den sçhon erwăhnten externae. Bei einer Missblldung der vorliegenden Art geht aber umgekehrt der rechte Venentheil, der sonst zur Vena cava sup. wird, unter, wăhrend der linke in seiner ganzen Länge bestehen bleibt. Das Verbindungsstück zwischen den beiderseitigen Venenanlagen ist ebenfalls ausgebildet, stellt aber nicht die Vena anonyma sin., sondern die Vena anonyma dextra dar. -

Es ist nach diesen kurzen Auseinandersetzungen wohl verständlich, dass das unterste Ende der Vena cava sup. sin. an der Stelle des Sin. coron. verlaufen muss. -

In unserem Falle sind noch einige andere Missbildungen bemerkenswerth, die in den früheren Fällen nicht mit erwähnt sind, die mir aber gerade für die Erklärung des Zustandekommens dieser anscheinend so willkürlichen Umkehrung normaler Verbältnisse bemerkenswerth erscheinen, ich meine die Aplasie der Gegend des Sinus transversus dexter bei Synostose ${ }^{\mathbf{l}}$ ) der Sutnra mastoidea und das Fehlen der Vena jug. int. dextra. Man wird ja keinesfalls annehmen können, dass diese

1) oder genauer ausgedrückt: frühzeltiger Verschmelzung der Anlagen des rechten Seitentheils rom Hinterhauptsbein und des Warzentheils vom Schläfenbein. 
letzterwähnten Missbildungen die Folge einer abnormen Persistenz des linken Duetus Cuvieri sind, während es mir sehr wahrscheinlich erscheint, dass der Mittelpunkt des ganzen Prozesses in der Synostose der Sutura mastoldea liegt.

Dass diese Synostose schon in früher Zeit entstanden ist, geht aus der beträchtlichen Aplasie des Schädels hervor, die in der Umgebung der ursprünglichen Nathstelle eingetreten ist. Es steht nun gewiss nichts im Wege anzunehmen, dass schon vor dem 3. oder 4. Monat des Embryonallebens (so lange bestehen die beiden Ductus Cuvieri beim Menschen) eine Verschmelzung der beiden benachbarten Knochenanlagen eintrat, durch welche eben ein mangelhaftes Wachsthum an der Stelle, wo sonst die Naht liegt, bedingt wurde. Bei dieser mangelhaften Ausbildung dieser Schädelpartie wurde vor allem auch das Foramen jugulare und der Sulcus und Sinus transversus dexter mit betroffen, die anmittelbar der Sutura mastoidea benachbart liegen, resp. sogar von ihr schief durchsetzt werden. Die Folge davon war dann weiter, dass das Blut nur in ungenügender Menge durch das Foramen jugulare dextrum hindurchtreten konnte und seinen Hauptweg aus dem Schädel durch den linken Sinus transversus und das linke Foramen jugulare zu nehmen gezwungen war.

Auch der Sulcus longitudinalis sup. setzt sich hier nicht, wie gewöhnlich, in den rechten, sondern in den linken Sulcus transversus fort. Da nun die Vena jugularis interna ein secundäres Gebilde ist, so ist es ferner verständlich, dass es hier auf der rechten Seite gar nicht zur Bildung einer solchen kam, da wohl die Vena jug. primitiva (die spätere Jugularis externa) zur Abführung dieser verhältnissmässig geringen Blutmasse genügte ${ }^{2}$ ).

Eine weitere Folge der mangelhaften Entwickelung des Sinus transvers. dexter und der übermässigen des S. sinister war nun die, dass der linke D uctus Guvieri verhältnissmässig viel mehr Blut bekam, als der rechte, während normal dłe Verhältnisse wohl umgekehrt gedacht werden müssen, und dass demnach der linke Dactus Cavieri nicht nur persistirte, sondern auch für die Fortschaffung des Blutes vollkommen ausreichte, so dass der rechte der obliteration anheim fiel.

Es sel nur besonders noch darauf aufmerksam gemacht, dass eine solche Synostose der Sutura mastoidea eine Missbildung der vorliegenden Art nur dann bewirken kann, wenn dieselbe vor dem 3. Embryonalmonat erfolgt.

2) Auf welche Weise die Communication vom For. jugulare mit den Aesten der Vena jug. externa erfolgte, konnte allerdings in unserem Falle nicht untersucht werden, aber gerade an dieser Stelle bestehen ja durch die Venae faciales auch unter normalen Verbältnissen genügende Anastomosen zwischen den Gebieten der Vena jug. ext. et int.; ansserdem gingen durch ein grosses For. mastoideum und einen weiten Canalis condyloideus Verbindungswege vom Sinus transvers. dexter zur Schädeloberfläche. 